\title{
Anti-uniform semilattices
}

\section{J. M. Howie and B. M. Schein}

\begin{abstract}
An inverse semigroup which is a union of groups is called Cliffordian. A semilattice $E$ is called universally Cliffordian if every inverse semigroup having $E$ as semilattice of idempotents is Cliffordian. It is shown that $E$ is universally Cliffordian if and only if it is anti-uniform, that is, if and only if no two distinct principal ideals of $E$ are isomorphic.

A semilattice $E$ satisfying the minimum condition is anti-uniform if and only if it is a well-ordered chain. Examples are given of anti-uniform semilattices of more complicated types.
\end{abstract}

A.H. Clifford [2] has given a complete description (in terms of groups and semilattices) of the structure of inverse semigroups that are unions of groups, and for this reason we shall (as in [7]) refer to such inverse semigroups as Cliffordian. If $E$ is a given semilattice, there do of course exist Cliffordian inverse semigroups having $E$ as semilattice of idempotents, the simplest such being $E$ itself. Let us temporarily call a semilattice $E$ universally Cliffordian if every inverse semigroup having $E$ as semilattice of idempotents is Cliffordian. The content of Theorem 7.5 in [3] is that $E$ is universally Cliffordian if it is finite and forms a chain under its natural ordering. More generally, one of us [7] has shown that $E$ is universally Cliffordian if it is a well-ordered chain (under its natural ordering).

It was conjectured in [7] that the converse also holds: $E$ is universally Cliffordian only if it is a well-ordered chain. The purpose of this note is to show that this conjecture is correct provided we restrict attention to semilattices with minimum condition. In general the conjecture

Received 21 March 1969. Received by J. Austral. Math. Soc. 28 October 1968. Revised 20 January 1969. Communicated by G.B. Preston. 
is false.

We are indebted to Professor W.D. Munn for the illustrated example in $\$ 2$ and for a number of other helpful suggestions.

\section{Anti-uniformity}

Various basic definitions and results of semigroup theory, all to be found in $\mathrm{Clifford}$ and Preston [3], will be used without comment.

If $E$ is a semilattice, we define an equivalence relation $U$ on $E$ by

$$
(e, f) \in U \text { if and only if } E e \cong E f \text {. }
$$

If $U$ is the identical relation on $E$ we shall say that $E$ is anti-uniform (a term suggested by Munn's use of 'uniform' for semilattices in which $U$ is the universal relation [6]).

If. $S$ is an inverse semigroup having $E$ as its semilattice of idempotents, and if $D$ denotes Green's equivalence, then $(e, f) \in D \cap(E \times E)$ if and only if there exists $a$ in $S$ such that $a a^{-1}=e$ and $a^{-1} a=f$. (See, for example, the proof given of Lemma 8.34 in [3].) In this case, as remarked in [4], there is an isomorphism $\alpha_{a}: E e \rightarrow E f$ defined by

$$
x \alpha_{a}=a^{-1} x a \quad(x \in E e)
$$

Thus

$$
D \cap(E \times E) \subseteq U \text {. }
$$

Now, an inverse semigroup is Cliffordian if and only if $a a^{-1}=a^{-1} a$ for every $a$, that is, if and only if

$$
D \cap(E \times E)=\iota_{E} \text {, }
$$

the identical relation on $E$. Thus we have established half of the following theorem, which reduces our problem to one purely in the theory of partially ordered sets.

THEOREM 1. A semilattice $E$ is universally Cliffordian if and only if it is anti-uniform. 
Proof. To establish the remaining half, we shall show that if $E$ is not anti-uniform then there exists a non-Cliffordian inverse semigroup having $E$ as its semilattice of idempotents. To do this, we use a construction due to Munn [6]. For each $(e, f)$ in $u$, let $T_{e, f}$ be the set of all isomorphisms from $E e$ onto Ef, and let

$$
\left.T_{E}=U T_{e, f}:(e, f) \in U\right\} \text {. }
$$

Munn has shown [6, Lemma 2.2] that, under the usual multiplication of one-one partial mappings, $T_{E}$ is an inverse semigroup. Moreover, the set of idempotents of $T_{E}$ is $E^{*}=\left\{\varepsilon_{e}: e \in E\right.$, where $\varepsilon_{e}$ is the identical mapping of $E e$ onto itself. Since $\varepsilon_{e} \varepsilon_{f}=\varepsilon_{e f}$ it is possible to identify $\varepsilon_{e}$ with $e$ and to say that $E$ is the semilattice of idempotents of $T_{E}$.

If $E$ is not anti-uniform, there exists $\alpha \in T_{e, f} \subseteq T_{E}$ with $e \neq f$. Clearly $\alpha \alpha^{-1}=e, \alpha^{-1} \alpha=f$, and so $T_{E}$ is not Cliffordian. This completes the proof.

We can now prove the conjecture in [7] in the case where $E$ has the minimum condition (by which we mean that every non-empty subset of $E$ has at least one minimal element).

THEOREM 2. If a semilattice has the minimum condition and is anti-uniform, then it is a welz-ordered chain.

Proof. We show (what is clearly sufficient) that if $E$ is not totally ordered, then it is not anti-uniform.

Let us define a subset $K$ of $E$ by saying that $x \in K$ if there exist elements of $E$ that are incomparable with $x$. Clearly $K \neq \phi$ if $E$ is not totally ordered; let $e$ be a minimal element of $K$, and let $f$ be a minimal member of the non-empty set of elements of $E$ that are incomparable with $e$. Then $e f<e$ and $e f<f$, since orherwise $e$ and $f$ would be comparable. In fact $e$ covers ef, for if $g$ is such that $e f \leqq g<e$, then $f e f \leqq f g \leqq f e$ and so $f g=e f$. But $g$ must be comparable with $f$, and so $f g=f$ or $f g=g$. The former alternative leads to $f=e f$, a contradiction; the latter alternative leads to $g=e f$. Thus $e$ covers $e f$, and similarly $f$ covers $e f$. It follows that 


$$
E e=E \text { ef } \cup\{e\}, \quad E f=E \text { ef } \cup\{f\},
$$

and so $(e, f) \in U$, an obvious isomorphism from Ee onto Ef being that which associates $e$ with $f$ and every other element with itself. This completes the proof.

\section{Examples}

We describe first a countable totally ordered set $E$ which is anti-uniform but not well-ordered. The set in question has been described by Anne C. Morel [5, p.70] and is quoted by Chang and Ehrenfeucht [1, p.143], the property of interest there being not dissimilar to the property of anti-uniformity. ${ }^{1}$

Consider the set $Q$ of rational numbers, and let $\varepsilon$ be any injection of $Q$ into $N=\{0,1,2, \ldots\}$. Let

$$
E=\underset{q \in Q}{U}(\{q\} \times\{0,1, \ldots, \varepsilon(q)\}),
$$

and define an order relation $\leqq$ on $E$ lexicographically:

$$
(q, m) \leqq(r, n)
$$

if and only if either $q<r$ or $q=r$ and $m \leqq n$.

Note that the set of elements of $E$ having no immediate predecessors is $\{(q, 0): q \in Q\}$, and the set of elements having no immediate successors is $\{(q, \varepsilon(q)): q \in Q\}$.

To show that $E$ is anti-uniform, suppose, by way of contradiction, that there exist distinct elements $(q, m),(r, n)$ in $E$ for which there is an isomorphism $\phi: E(q, m) \rightarrow E(r, n)$. We distinguish two cases:

(i) $m \neq n$;

(ii) $m=n$ and $q \neq r$.

In case (i), certainly

$$
(q, m) \phi=(r, n),(q, m-1) \phi=(r, n-1), \ldots \ldots .
$$

If, without essential loss of generality, we suppose that $m>n$, we eventually find that

\footnotetext{
${ }^{1}$ We are indebted to $\mathrm{Dr}$ J.N. Crossley for these references.
} 


$$
(q, m-n) \phi=(r, 0),
$$

a contradiction, since $(q, m-n)$ has an immediate predecessor and $(r, 0)$ does not.

In case (ii) we may suppose without loss of generality that $q>r$. If $s$ is such that $r<s<q$, then $(s, 0) \in E(q, m)$, and has no immediate predecessor; hence $(s, 0) \phi \in E(r, n)$, and has no immediate predecessor:

that is, $(s, 0) \phi=(t, 0)$, where $t \leqq r$. Certainly $t \neq s$ and so $\varepsilon(t) \neq \varepsilon(s)$. We shall consider only the case in which $\varepsilon(s)>\varepsilon(t)$, since the other case is similar. Now

$$
(s, 1) \phi=(t, 1),(s, 2) \phi=(t, 2) \ldots \ldots,
$$

and finally $(s, \varepsilon(t)) \phi=(t, \varepsilon(t))$, a contradiction, since $(s, \varepsilon(t))$ has an immediate successor and $(t, \varepsilon(t))$ does not.

The set $E$ is of course not well-ordered, since for each $q$ in $Q$ the non-empty subset $\{x \in E: x>(q, \varepsilon(q))\}$ does not have a least element.

An anti-uniform semilattice need not be totally ordered. If, for example, we take the union of the semilattice $E$ described above with the semilattice $N=\{0,1,2,3, \ldots\}$ under the natural ordering, and define $e n=0$ for all $e$ in $E$ and $n$ in $N$, we obtain an anti-uniform semilattice. The diagram illustrates an example of an anti-uniform semilattice satisfying the maximum condition, suggested to us by Munn.

We are unable to give a complete classification of anti-uniform semilattices.

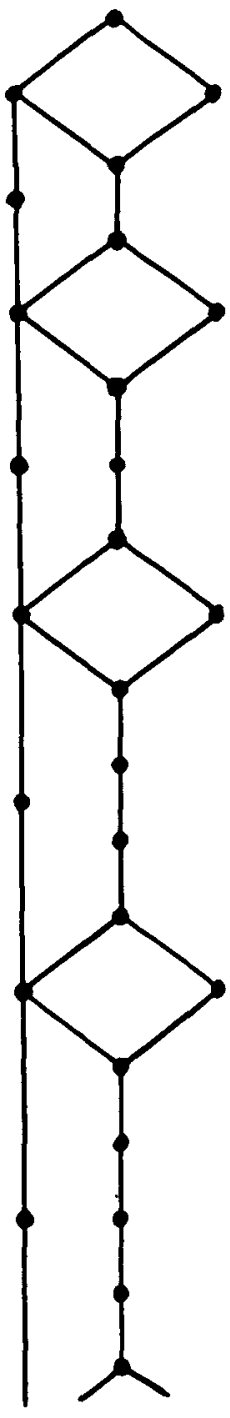

etc. 


\section{References}

[1] C.C. Chang and A. Ehrenfeucht, "A characterisation of abelian groups of automorphisms of a simply ordering relation", Fund. Math. 51 (1962), 141-147.

[2] A.H. Clifford, "Semigroups admitting relative inverses", Ann. of Math. (2) 42 (1941), 1037-1049.

[3] A.H. Clifford and G.B. Preston, The algebraic theory of semigroups, vols. 1 and 2, (Amer. Math. Soc. Mathematical Surveys 7, Providence, 1961 and 1967).

[4] J.M. Howie, "The maximum idempotent-separating congruence on an inverse semigroup", Proc. Edinburgh Math. Soc. (2) 14 (1964), 71-79.

[5] Anne C. Morel, "On the arithmetic of order types", Trans. Amer. Math. Soc. 92 (1959), 48-71.

[6] W.D. Munn, "Uniform semilattices and bisimple inverse semigroups", Quart. J. Math. Oxford. (2) 17 (1966), 151-159.

[7] B.M. Schein, "Generalized groups with the well-ordered set of idempotents", Mat.-Fyz. Casopis Sloven. Akad. Vied. 14 (1964), 259-262.

University of Stirling,

stirling, Scotland,

and

University of Saratov,

Saratov, USSR. 